\title{
Experiences with Wet Capsule Imaging Exploring the Potential for Live Cell Imaging
}

\author{
C.A. Ackerley, ${ }^{*}$ C. Nielsen, ${ }^{* *}$ and C.E. Hawkins* \\ * Division of Pathology, Hospital for Sick Children, 555 University Ave.,Toronto, ON. M5G1X8 \\ ** JEOL USA, 11 Dearborn Road, Peabody, MA 01960
}

\begin{abstract}
Although there are many light microscopic methods for imaging live cells, they do not have the resolution of electron microscopy. Significant efforts have been put into the development of scanning electron microscopes (SEM) which are capable of imaging wet samples, thus eliminating many of the artefacts associated with traditional sample preparation. This has yet to be achieved in life science applications however adaptations to SEMs for the observation of partially hydrated materials have been developed. Technological improvements to achieve this goal include the development of variable pressure columns and electron detectors that are efficient when imaging samples in a vapour environment (environmental SEM, ESEM). Using this approach, imaging of cells and tissues at acceptable resolution and obtaining adequate contrast is questionable [1].
\end{abstract}

A new technology has been developed allowing cells grown on an electron lucent partition membrane to be stained using either one of a number of protocols including conventional electron microscopy fixation and staining, cytochemistry and immunogold immunocytochemistry. The cells are then sealed in a capsule that contains a liquid imaging media that dissipates electrons and provides some thermal insulation. This capsule maintains a pressure of one atmosphere and the cells can be observed using any SEM equipped with a backscatter electron (BE) detector. Thus unlike ESEM and similar techniques, the sample is completely isolated from the vacuum within the microscope. The images themselves are dependent upon a number of factors including accelerating voltage, probe current and backscatter electron (BE) detection efficiency. This wet SEM technique uses BEs the majority of which originate from the portions of the cells that are adhered to the partition membrane of the chamber. Although an electron scanning reflectance mode is used, the image is generated from material within the cells that yield significant atomic contrast. Subcellular organelles can be distinguished based on differences in local concentration of lipids and salts within biological samples and a wide variety of stains and labels can be used to enhance contrast [2].

In a series of preliminary experiments, both pseudomonas aeruginosa bacteria and an astrocytoma derived cell line (U343MG) were grown on the partition membrane of the capsules. The cells were fixed with glutaraldehyde, stained with uranyl acetate and sealed in the capsules immersed in imaging buffer. They were then imaged in a field emission SEM (FESEM) using a conventional solid state BE detector. Bacterial flagella were easily distinguished as well as structures that looked like pili in the pseudomonas samples. In the capsules that contained the astrocytoma derived cells, cell filipodia, cytoskeleton and nuclei were easily distinguished. Some of the astrocytoma cells grown on the partition membrane were fixed in paraformaldehyde and immunogold labelled with $30 \mathrm{~nm}$ colloidal gold bound to an antibody against CD44 and sealed in the capsules immersed in imaging buffer. When imaged in the FESEM the cells were heavily decorated with gold particles. 
We then imaged both live bacterial cells and immunogold labeled live cells. Cells were grown in imaging buffer reconstituted with the appropriate tissue culture media on the partition membrane and incubated until the cells were established. Once the astrocytoma cell lines had grown CD44 antibody gold complexes were added at the appropriate concentration. Both cell cultures were then examined in the FESEM and maintained at a constant $37^{\circ} \mathrm{C}$ during the examination of these cells. Images were obtained using a conventional solid state BE detector. Cells were then assessed under the light microscope for any obvious electron beam damage. Immediately following electron beam exposure some of the capsules containing astrocytoma cells were also assessed for damage using Trypan Blue exclusion.

Although solid state BE detectors have the best $\mathrm{Z}$ contrast they must be used at slow scan rates. In both the bacterial and astrocytoma cell cultures no observable damage was seen as long as electron beam dwell time was minimized. Immunogold label was easily detected on the astrocytoma cell lines (fig.1). As the cells were alive some motion was detected either due to cell motility or irritation by the electron beam during the slow exposure used during image recording (fig.2). Trypan Blue exclusion results were similar to unexposed cell cultures. Astrocytoma cells in capsules that were opened and placed back in the incubator overnight were all confluent the following day.

In order to overcome some of the motility issues and minimize electron beam exposure damage we are currently modifying our microscope. Optimum electron beam conditions will be determined using a trial and error approach and monitoring the conditions with a picoammeter. Dwell time will be minimized by beam blanking between exposures. A YAG photomultiplier based BE detector has been installed and the near TV rate signal will be captured by a scan converter. Serial time lapse images of the same object will be recorded. It is hoped that using this approach we will be able to capture dynamic cell processes including cell to cell interactions, cell to extracellular matrix interactions and drug effects.

\section{References}

[1] G. Danilotis, J. Microsc162 (1991) 391.

[2] S. Thiberge et al., Proc Natl Acad Sci USA 10 (10) (2004) 3346.

[3] This work was funded in part by a NIH SCOR grant to CAA
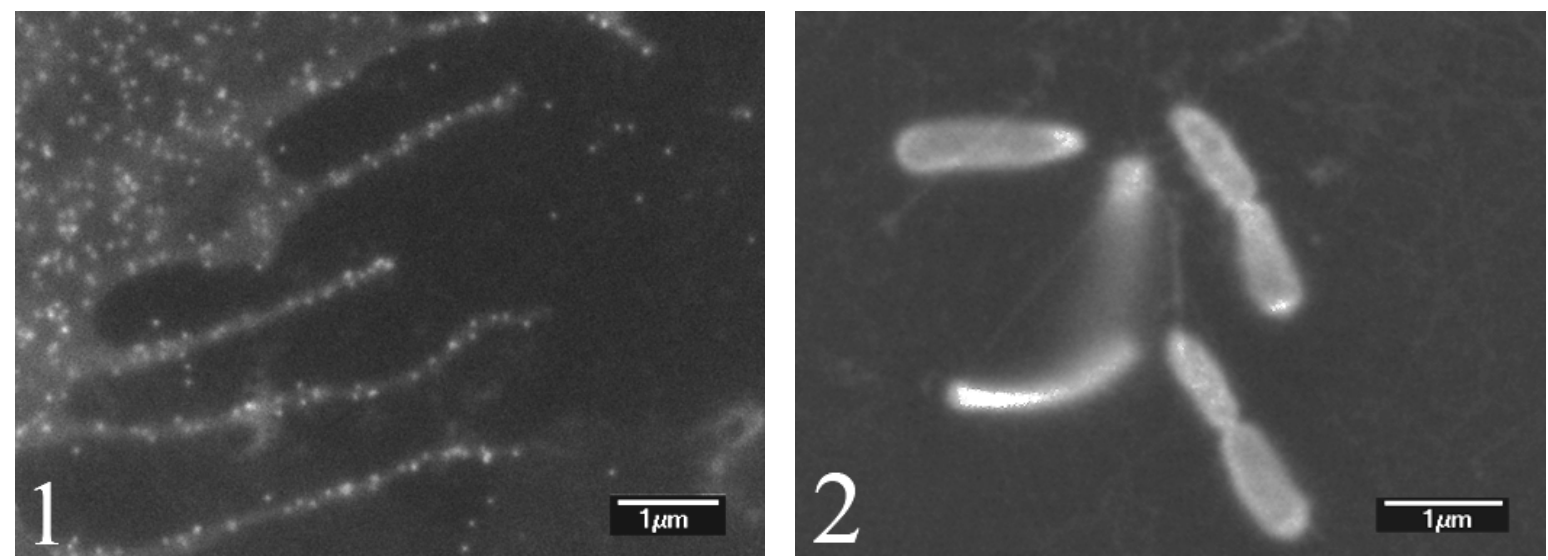

Fig.1 Live astrocytoma cell that has been immunogold labeled for CD44.

Fig. 2 Live pseudomonas aeruginosa cells imaged with a solid state BE detector in a wet cell. Note the movement in some of the cells as the image was acquired. 